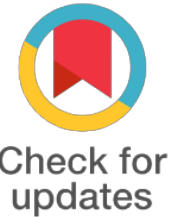

*For correspondence:

thilathy@upm.edu.my

Competing interests: The authors declare that no competing interests exist.

Received: 2017-07-11

Accepted: 2017-08-15

Published: 2017-09-05

Copyright The Author(s) 2017. This article is published with open access by BioMedPress (BMP).

This article is distributed under the terms of the Creative Commons Attribution License (CC-BY 4.0) which permits any use, distribution, and reproduction in any medium, provided the original author(s) and the source are credited.

\section{Animal Component Free Medium Retains the Stability of Hypervariable Region 1 (HV1) of Mitochondrial DNA of Human Full Term Amniotic Fluid Mesenchymal Stem Cells}

ORAL

\author{
Rohayu Izanwati Mohd Rawi, Sharizah Alimat , Norshariza Nordin , Zulida Rejali , \\ Thilakavathy Karuppiah
}

Department Of Biomedical Sciences, Faculty of Medicine and Health Sciences, Universti Putra Malaysia (UPM), Serdang, Selangor Darul Ehsan 43400, Malaysia

\section{Abstract}

Therapeutic potential of human amniotic fluid (hAF) cells in treating human disease is very promising. Furthermore it provides an alternative in isolating stem cells from an accessible source, where the fluid is merely discarded, thus circumventing the ethical concerns. Mesenchymal stem cell (MSC) is one of the heterogenous cell populations in the amniotic fluid $(\mathrm{AF})$, however, its role and potential for stem cell therapy are yet to be discovered. One of the criteria in establishing the clinical grade of MSCs is the ability of the cells to retain their genomic stability in vitro prior to bedside applications. The microenviroment niche of the culture medium used is important to generate the safe MSCs. As mitochondria play the vital role in metabolic task such as apoptosis and cell proliferation, therefore investigating mitochondria DNA (mtDNA) is essential to mark the stability of stem cell. In this study, we aimed to detect variations of mtDNA across the passage number of cultured human amniotic fluid MSCs (hAFMSCs), cultured in animal component free (ACF) medium, specialised for human MSCs. Polymorphisms of hypervariable region 1 (HV1) in the D-loop of mtDNA were investigated in a series of cultured hAFMSCs (passage 1 and 3 ) from three different samples (three biological replicates) as well as in their respective fresh samples (control). Extracted DNA samples were subjected to PCR to detect the sequence variations of HV1 (nt16024-16365). The sequences were then analysed using SeqScape v2.5 by comparing the sequences with The Cambridge Reference Sequence (rCRS) for human mtDNA. The common C-to-T transition at position 16223 (C16223T) for all three samples was identified. Another common base transition was also identified for the other two samples at position 16311 (T16311C). However, one of the samples showed more variants; a C-to-T transition at position 16290 (C16290T), an A-to-G at position 16317 (A16317G) and a G-to-A at position 16319 (G16319A). All variants were identified within the sequence of around 341bp nucleotides and their polymorphisms frequency was consistent for all three samples regardless of their passage numbers when compared to control. This pattern of polymorphisms demonstrates the HV1 mtDNA integrity of hAFMSCs cultured in ACF medium, hence highly suggesting the ability of ACF medium in retaining the stability of stem cells mtDNA in vitro. The ACF medium may serve as a good medium in culturing hAFMSCs that is safe and stable for basic research in stem cell and therapeutic applications. 


\section{Biomedical \\ Research \& Therapy}

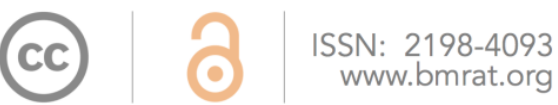

Keywords

Human Amniotic Fluid, Mesenchymal Stem Cells, mtDNA, Polymorphisms, Animal Component Free Medium.

Funding

FRGS (5524538)

References 